\title{
The impact of parenteral opioid analgesics on sonographic and clinical diagnostic accuracy for suspected acute appendicitis
}

\section{Clinical question}

In patients with suspected appendicitis, does the administration of opioid analgesics alter the sonographic or clinical diagnostic accuracy?

\section{Article chosen}

Vermeulen B, Morabia A, Unger PF, Goehring C, Grangier C, Skljarov I, et al. Acute appendicitis: influence of early pain relief on the accuracy of clinical and US findings in the decision to operate. A randomized trial. Radiology 1999;210:639-43.

\section{Objective}

To determine whether the administration of opioid analgesics influences sonographic diagnosis or surgical decision-making in cases of suspected acute appendicitis.

\section{Background}

Surgeons have long opposed the early use of opioid analgesics in patients with abdominal pain, fearing that these agents will mask the physical signs of a surgical abdomen and lead to delayed or missed diagnosis. This belief has been refuted by several studies, which show that diagnostic accuracy is the same ${ }^{1-3}$ or better ${ }^{4}$ in patients who receive opioid analgesia.

\section{Population studied}

Patients over age 16 who presented to the emergency department (ED) with right lower quadrant pain were eligible for the study unless they had one of the following exclusion criteria: previous appendectomy, clinical presentation consistent with nonappendicular condition, renal, hepatic, or respiratory insufficiency, or the use of psychotropic medication. Of 488 eligible patients, 350 (72\%) consented and were enrolled in the study. Ten were excluded -7 because clinical or radiological information was missing, 2 because of loss to follow-up at 1 month, and 1 because the operation took place before the protocol was completed. Outcomes of patients who refused to participate were not reported.
Reviewers: Michael Bullard, MD;* Sunil Sookram, MD;* Bruce Campana, MD $\dagger$

*Division of Emergency Medicine, University of Alberta, Edmonton, Alta.; †King Faisal Specialist Hospital \& Research Center, Riyadh, Saudi Arabia.

Date appraised: July 31, 1999

\section{Study design}

This was a randomized, double-blind clinical trial of singledose intravenous morphine $(0.1 \mathrm{mg} / \mathrm{kg}$ over 5 minutes $)$ versus saline placebo. Visual analog (VAS) pain scores were recorded prior to, and 45 minutes after study drug administration. Radiology residents performed abdominal ultrasound (U/S) within 4 hours of drug administration. Following U/S, surgeons used clinical findings, laboratory results and the sonographic examination to decide whether to perform surgery. Final diagnosis was confirmed histologically in the operative group and by 1-month clinical follow-up in the non-operative group.

\section{Outcomes measured}

The primary outcome was U/S diagnostic accuracy in patients receiving morphine versus those receiving placebo. VAS pain scores and the clinical accuracy ("appropriateness") of the surgeons' decision whether or not to operate were reported for both groups.

\section{Results}

Overall, 175 patients received morphine and 165 received placebo. Of these, 205 underwent laparotomy and 171 had a final diagnosis of appendicitis. Ultrasound sensitivity was lower $(71.1 \%$ vs. $80.6 \% ; p=0.05)$ and specificity was higher $(65.2 \%$ vs. $53.8 \% ; p=0.05)$ in the morphine group, but there was no difference in the accuracy of surgical decisionmaking, regardless of whether patients received analgesics. Surgery was appropriate in $83.7 \%$ of placebo recipients vs. $83.2 \%$ of morphine recipients $(p=\mathrm{NS})$. All discharged 
patients in both groups were discharged appropriately and there were no delayed or missed diagnoses of appendicitis at 1-month follow-up.

\section{Study conclusions}

Opioid analgesia did not improve the diagnostic performance of U/S and had no apparent effect on the surgeons' ability to identify surgical pathology.

\section{Commentary}

This study is interesting from 2 perspectives. First, it suggests that opioid analgesia does not increase the risk of delayed or missed diagnosis of appendicitis and does not influence the rate of unnecessary laparotomy. Second, it suggests that U/S is less sensitive, less specific, and less accurate than clinical judgement. In this study, the positive and negative likelihood ratios for U/S were, respectively, 1.87 and 0.45 . A test with these parameters is both a weak negative and a weak positive predictor. For example, given a hypothetical "grey-zone" patient with pre-test probability of $50 \%$, a negative U/S would decrease (post-test) probability to approximately $30 \%$, while a positive U/S would increase (post-test) probability to only $65 \%$. In both cases, this offers little help to the clinician. These data suggest that greater dependence on U/S in this setting is likely to increase, rather than decrease diagnostic error.

These results are consistent with 3 other studies, ${ }^{1-3}$ which found no change in diagnostic accuracy or morbidity in patients receiving opioids; however, they contrast slightly with a fourth study, which also reported no missed diagnoses among opioid recipients, but found an increased rate of unnecessary laparotomy in the placebo group. The authors of this study ${ }^{4}$ calculated a number-needed-to-treat (with opioids) of 8.33 to prevent a negative laparotomy.

While all 5 studies combined include just over 600 patients, none have shown a trend toward missed diagnoses or significant diagnostic delays in patients receiving opioid analgesics. Therefore, the weight of the evidence supports judicious analgesic use in ED patients being evaluated for suspected surgical abdominal pain.

\section{References}

1. Zoltie N, Cust MP. Analgesia in the acute abdomen. Ann R Coll Surg Engl 1986;68:209-10.

2. Pace S, Burke TF. Intravenous morphine for early pain relief in patients with acute abdominal pain. Acad Emerg Med 1996; 3:1086-92.

3. LoVecchio F, Oster N, Sturmann K, Nelson L, Flashner S, Finger $\mathrm{R}$. The use of analgesics in patients with acute abdominal pain. J Emerg Med 1997;15:775-9.

4. Attard AR, Corlett MJ, Kidner NJ, Leslie AP, Fraser IA. Safety of early pain relief for acute abdominal pain. BMJ 1992;305:554-6.

Correspondence to: Dr. Michael Bullard, Division of Emergency Medicine, University of Alberta Hospital, WMC 1G1.50, 8440112 St., Edmonton AB T6G 2B7

\title{
Peer Reviewers • Réviseurs
}

\begin{abstract}
Abu-Laban, Riyad
Allen, Tim

Berringer, Ross

Beveridge, Robert

Brubacher, Jeffrey

Cass, Dan E.

Chopra, Anil

Christenson, James

Cummings, Garnet

Cummings, Greta

Deady, Brian

Dickinson, Garth
\end{abstract}

\author{
Dubinsky, Isser \\ Fernandes, Chris \\ Frank, Jason R. \\ Gareau, Annie \\ Howlett, Michael \\ Huber, Alan \\ Innes, Grant \\ Ip, Urbain \\ Joubert, Gary \\ Lee, Jacques \\ Letovsky, Eric \\ Lexchin, Joel
}

CJEM would like to recognize and thank the following peer reviewers who have volunteered their time, knowledge and effort to CJEM. Peer review is essential to medical journal's credibility and success.

This list includes the peer reviewers who helped us from November 1998 to August 1999. We apologize in advance if we have overlooked a reviewer, but please let us know so we can acknowledge you in a future issue.

Lucky, Cindy-Ann

MacPhail, Iain

Mann, David

McGuire, Peter

McKnight, Doug

Minnes, Bruce

Morrison, Laurie J.

Mosdossy, Gregory

Nijissen-Jordon, Cheri

O'Connor, Michael

Petrie, David

Ross, John
Schull, Michael J.

Shuster, Michael

Sinclair, Doug

Spence, Julie

Tallon, John M.

Taylor, Anthony S.

Thompson, James

Verbeek, Richard

Vertesi, Les

Ward, John 